\title{
Temporal Analysis of Biometric Template Update Procedures in Uncontrolled Environment
}

\author{
Ajita Rattani, Gian Luca Marcialis, and Fabio Roli \\ Department of Electrical and Electronic Engineering \\ University of Cagliari, Italy \\ \{ajita.rattani, marcialis, roli\}@diee.unica.it
}

\begin{abstract}
Self-update and co-update algorithms are aimed at gradually adapting biometric templates to the intra-class variations. These update techniques have been claimed to be effective in capturing variations occurring in medium time period but no experimental evaluations have been done in the literature to clearly show this fact. The aim of this paper is the analysis and comparison of these update techniques on the sequence of input batch of samples as available over time, specifically, in the time-span of 1.5 years. Effectiveness of these techniques have been compared in terms of capability to capture significant intra-class variations and the attained performance improvement, over time. Experiments are carried out on DIEE multi-modal dataset, explicitly collected for this aim. This dataset is publicly available by contacting the authors.
\end{abstract}

Keywords: Biometrics, Face, Fingerprint, Self-update,Co-update.

\section{Introduction}

A personal biometric verification system consists of two main processes; enrolment and matching. In enrolment, individual's biometric samples are captured, processed and features extracted. These extracted features are labelled with user's ID and is referred to as "template". Matching mode verifies claimed identity by comparing input sample(s) to the enrolled template(s) [1] 2].

The enrolment process typically acquires very few samples, usually a single image, captured under controlled conditions. On the other hand, real time operation in uncontrolled environment encounters large variations in the input data, called "intra-class" variations. These variations can be attributed to factors like human-sensor interaction, illumination conditions, changes in sensor, seasonal variations, occlusions due to user's accessories etc.. These factors causes "temporary variations" in the biometric data. In addition, biometric traits also undergo gradual ageing process as a result of time lapse [3]. Accordingly, changes in the biometric over time can be termed as "temporal changes" occurring in the medium-long term.

As a consequence of these temporary and temporal variations, enrolled templates becomes "un-representative". In this paper, we refer to representative 
templates as templates with the capability to correctly recognize significant intraclass and inter-class variations (impostor samples).

Recently, template update procedures have been introduced aiming to solve the issue of unrepresentative templates by constantly adapting themselves to the intra-class variations of the input data. Most of the existing template update techniques are based on self-update and co-update algorithms [4. In self-update, biometric system adapts itself on the basis of highly confidently classified input data [5]. Template co-update utilizes the mutual and complementary help of multiple biometric traits to adapt the templates to the variation of the input data [6].

Reference [7] performs experimental comparison of self-update and co-update proving the efficacy of co-update, on utilizing the help of multi-modalities, in capturing intra-class variations available over short-term period (e.g. face expression variations), over the former. However, the experimental analysis and comparison is performed [7] over a limited data set containing only temporary variations.

Till date, no analysis have been done for self and co-update techniques with respect to :

- the dependence of their performances on the representativeness of initial templates,over time;

- their efficiency in capturing intra-class variations as available over time;

- their resulting performance improvement over dataset containing both temporary as well as temporal variations.

To this end, the aim of this paper is to advance the state-of-the-art by a temporal analysis of self and co-update algorithm by using input batches of samples available at different time intervals. In particular, in this paper :

- we propose a conceptual representation of performance of self-update and co-update with respect to representativeness of the initial captured enrolled templates; this representation is supported by the experimental evidence.

- The capability to capture temporal intra-class variations and the obtained performances improvement have been analyzed over time.

- Experiments are conducted on DIEE multimodal dataset,explicitly collected for this aim, containing both temporary as well as temporal variations.

In section II, self-update and co-update techniques are discussed. Experimental evaluations are presented in Section III. Section IV concludes the paper.

\section{Self-update and Co-update Algorithms}

In this section, we elaborate on self-update and co-update algorithms together with the possible conceptual explanation of their operation. 


\subsection{Self-updating}

In self-updating procedure, matcher is trained on a initial set of enrolled templates, named $T$. A batch of samples, $U$, is collected during system's operations over a certain time [5]. Among all the samples in $U$, only those samples whose match score against the available templates exceeds a given updating threshold (i.e., highly confidently classified samples), thr*, are used for the adaptation process. This is done to reduce the probability of impostor introduction (false acceptance) into the updated gallery set. This procedure is presented in Algorithm 1.

However, using only highly confident samples may lead to inefficiency in capturing samples representing substantial variations [4. Thus, the efficiency is dependent on the representativeness of the initial enrolled templates. On the other hand, by relaxing the updating threshold, the system may become prone to classification errors (updating using impostor samples) [4] [7].

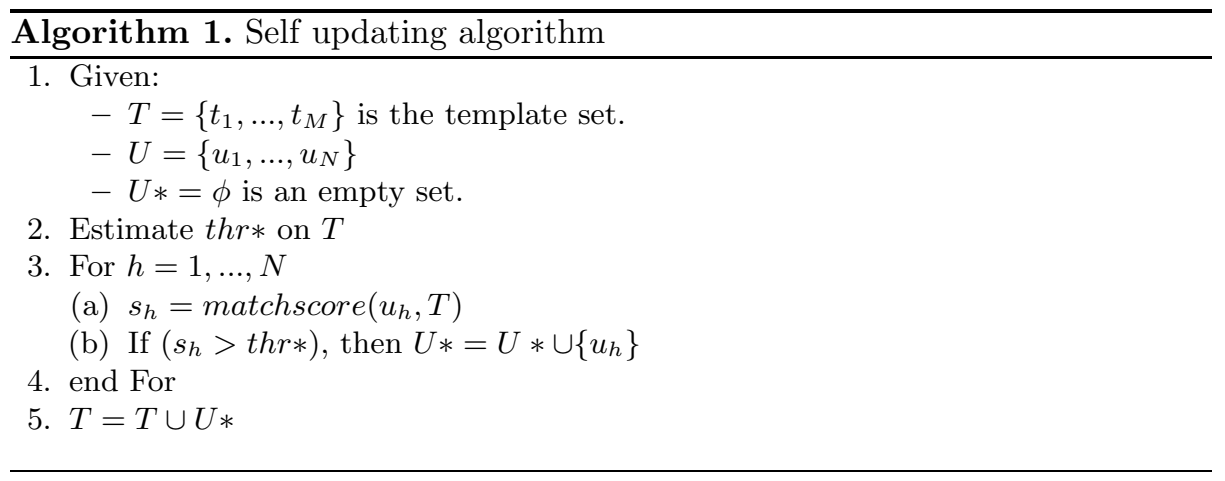

For the conceptual representation of self-update's operation, let us consider a sample space of a specific user for a given biometric $b 0$.

If this user-specific sample space is represented in the form of a directed relational graph $G$ where nodes are the samples and the edges are labelled with the related matching score between any two samples. On removing the edges labelled with the matching score below $t h r *$. The sample space may be partitioned into $n$ sub-graphs $G_{1}^{b 0}, \ldots, G_{n}^{b 0}$. Each of these subgraphs $G_{i}^{b 0}$, contains only those nodes (samples) connected to other nodes with edges labelled with matching score above thr*.

On the availability of input batch $U$, consisting of random samples forming the part of different subgraphs $G_{1}^{b 0}, \ldots, G_{n}^{b 0}$ in a user-specific sample space. Let us suppose for simplicity that only single initial enrolled template, $t$, is available, and belongs to subgraph $G_{1}^{b 0}$. Considering the self-update behaviour (Algorithm 1), may be only subset $U_{G_{1}^{b 0}}=\left\{u \in U: u \in G_{1}^{b 0}\right\}$ of samples belonging to subgraph $G_{1}^{b 0}$ may be inserted into the template gallery. Provided their exists a directed path from template $t$ to sample $u \in U\left\{G_{1}\right\}$. Other samples of $U \notin$ $U\left\{G_{1}\right\}$ will be completely neglected for the adaptation, in this case. 
This implies that, self-update will result in limited capture of samples depending on the representativeness of the enrolled templates. In other words, in order to allow self-update to be efficient in capturing significant variations, a careful a priori selection of templates must be done by human experts, on the basis of user-specific subgraphs characteristics, with respect to the given user population and the selected threshold thr*. Another alternative is to relax the threshold value,thr*, which changes the obtained subgraphs partitioning and reduces the number $n$ of subgraphs.

As an example, Figure 1(a), shows the hypothetical diagram with two component subgraphs $G_{1}^{b 0}$ and $G_{2}^{b 0}$ formed from the user-specific sample space, for any modality $b 0$. If the enrolled templates are in $G_{1}^{b 0}$, only samples lying in the subgraph $G_{1}^{b 0}$ can be captured by the self-update process. The samples in $G_{2}^{b 0}$ will be completely neglected for the adaptation process.

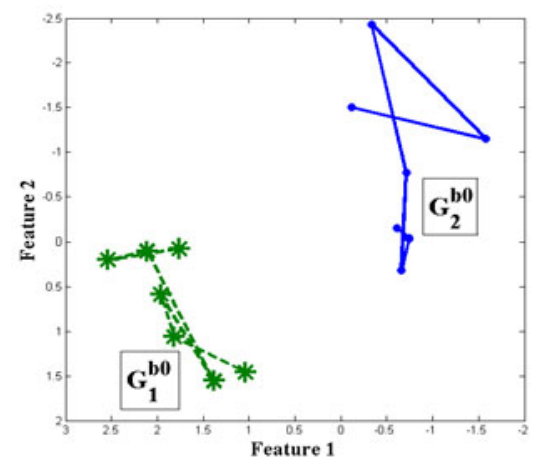

(a) Modality b0

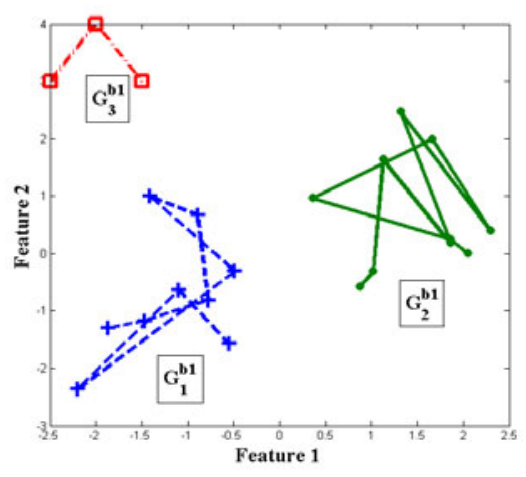

(b) Modality b1

Fig. 1. A hypothetical diagram showing the different component subgraphs $G^{b 0}$ and $G^{b 1}$, of a complete directional graph where nodes are the biometric samples hypothetically projected in the feature space, formed from removing the edges labelled with matching score below $t h r *$ for the modalities $b 0$ and $b 1$

\subsection{Template Co-updating}

In template co-update [6], mutual and complimentary help of two biometrics is utilized for the adaptation of the templates. Specifically, given two modalities, input samples classified as genuine with high confidence by the one modality (for ex, face), together with the corresponding complementary sample from the another modality (for ex, fingerprint), are used for the updating process.

Co-updating procedure is presented in Algorithm 2, where $T^{b 0}$ and $T^{b 1}$ are the template sets and $U^{b 0}$ and $U^{b 1}$ are the input batches of samples, for the 
modalities $b 0$ and $b 1$ (for example, fingerprint and face). The sample is highly confidently classified if its matching score on comparison with the enrolled templates is above the set threshold $t h r^{b 0} *$ and $t h r^{b 1} * . s_{h}^{b i}$ is the confidently classified sample by the modality $b_{i}$.

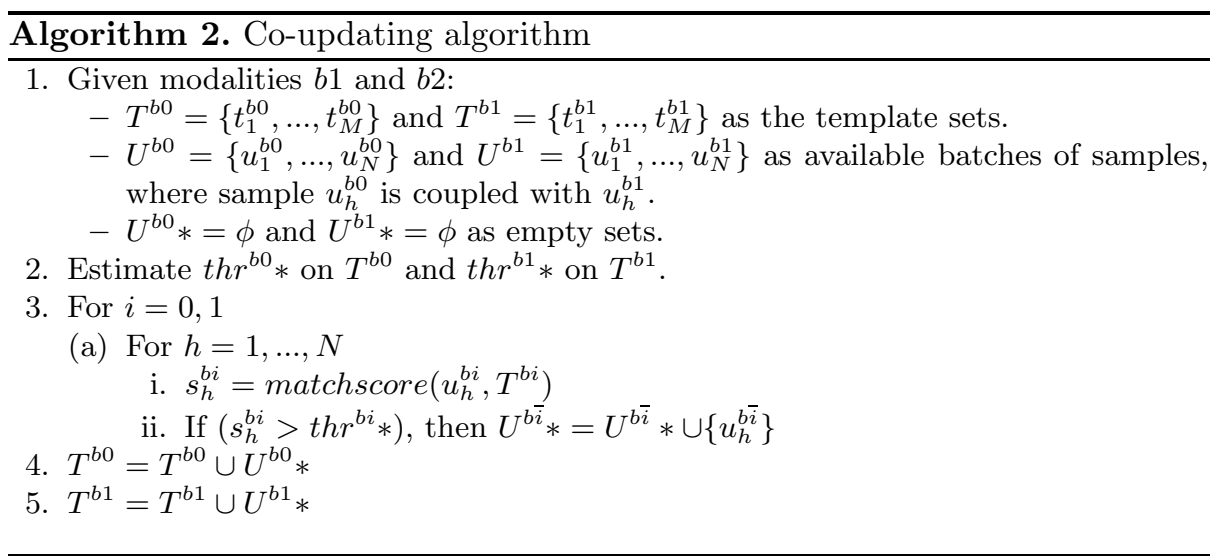

Following the same convention of directed relational graph and sub-graph partioning of the user-specific sample space, as used for the representation of self-update, the conceptual representation of co-update may be explained as well. Worth noticing that each modality may form different sub-graphs with different number of samples i.e, $G^{b 0}=\left\{G_{1}^{b 0}, \ldots, G_{n^{b 0}}^{b 0}\right\}$ and $G^{b 1}=\left\{G_{1}^{b 1}, \ldots, G_{n^{b 1}}^{b 1}\right\}$ where $n^{b 0} \neq n^{b 1}$. This is due to the difference in the match score distribution and complementary characteristics of different modalities. Figures $1(\mathrm{a})$ and $1(\mathrm{~b})$ show the component subgraphs of two independent modalities $b 0$ and $b 1$.

Irrespective of the induced partitioning (in the form of component subgraphs) of individual biometric modality, template galleries of each modality can be updated with the samples lying in the subgraphs different from the one in which the enrolled templates reside, using the complementary matcher. In other words, biometric $b 0$ uses biometric $b 1$ to update the template gallery, and vice-versa. This is done thanks to the conditional independence among two biometrics as assumed in co-updating algorithm: each sample of biometric $b 0, u^{b 0}$ may be coupled with any sample, $u^{b 1}$, of $b 1$, independently of user-specific sample space partitioning.

\section{Experimental Analysis}

\subsection{Data Set}

40 subjects with 50 samples per subjects are used from the DIEE multi-modal face and fingerprint dataset. These 50 samples are acquired in 5 sessions with 10 samples per session (batch) with a gap of minimum three weeks between 
two consecutive sessions. These batches are indicated as $B_{1}, \ldots, B_{5}$. The whole collection process span a period of 1.5 years. Each batch $B_{i}$ consist of fingerprint and face couples for a certain subject. For the co-update process these batches are used as it, however, for self updating process face and fingerprint samples are isolated. For the sake of simplicity, we indicated in the following mono- and multi-modal batches using the same notation.

For face biometrics, variations such as lighting, expression change (i.e., happy, sad, surprised and angry) and eyes half-closed are introduced in every batch. For fingerprint biometrics, rotation, changes in pressure, non-linear deformations and partial fingerprints are introduced as variations [8]. Two consecutive batches, $B_{i}$ and $B_{i+1}$ are temporally ordered. Figure 2 shows some of the images taken from different sessions of face modality for a randomly chosen subject, where images in different rows represent different sessions.

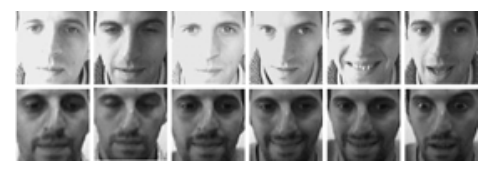

Fig. 2. Face images from different sessions exhibit ageing as well as other intra-class variations, for a randomnly chosen subject

\subsection{Experimental Protocol}

\section{- Training:}

1. The system is trained with 2 enrolled samples per user from the first session i.e, batch $B_{1}$.

2. Threshold for adaptation is set by estimating genuine distribution according to available templates.

- Updating: Remaining user images consisting of eight samples from the batch $B_{1}$ and ten samples from the remaining four batches i.e, $B_{2: 4}$, are used for adaptation as follows:

1. For each user, $B 1 \ldots B 4$ are available over time.

2. Each batch $B_{i}$ with $i \in 1: 4$ is used for updating the template set of the respective user by using the self-update and co-update algorithms as mentioned in Section II. These batches $B_{i}$ corresponds to unlabelled batch $U$ in algorithms 1 and 2 .

\section{- Performance evaluation:}

1. After updating using batch $B_{i}$, i.e., after updating cycle $i$, batch $B_{i+1}$ is used for testing the system performance. Scores for each test sample are always computed using the max rule [2].

2. At each updating cycle $i$, Equal Error Rate of the system, namely, $E E R_{i}$, is computed as follows: $E E R_{i}=\frac{i-1}{i} * E E R_{i-1}^{a v e}+\frac{1}{i} * E E R_{i}^{*}$, as the mean of the EER at previous update cycles i.e., $E E R_{i-1}^{a v e}$ and the EER $\left(E E R_{i}^{*}\right)$ obtained at the specific update cycle $i$.

3. After the evaluation of the system, the same batch $B_{i+1}$ is used for the process of updating. 


\subsection{Results}

The goals of these experiments are to:

- Provide experimental validation of the conceptual representation of the functioning of self-update and co-update as provided in section II. This is done by evaluating the capability of self and co-update techniques in capturing significant intra-class variations and its dependence on the representativeness of the initial enrolled templates over time.

- Evaluation of these techniques over time, following multiple update cycles, that is, using batches $B 1, \ldots, B 5$.

\subsection{Experiment no. \#1}

The aim of this experiment is to validate the dependence of self-update on initial templates and to verify the contrary for co-update in capturing large intra-class variations, according to the conceptual representation of Section II.

For sake of space, we present results only for the face biometric, but similar results may be obtained for the fingerprint biometrics as well.

In confirmity with the conceptual representation in section II, structure of data is studied and following steps have been performed, considering all the samples for each user:

- The graph $G$ is formed and partitioned by connecting each sample to only those samples with matching score above the acceptance threshold set at $0.001 \%$ FAR of the system. This is a very stringent value in order to avoid presence of impostors in the graph.

- Then, two samples are chosen as templates from the subgraph containing batch $B_{1}$ i.e, $G^{\text {face }}(B 1)$, for face modality.

- Self- and co-update algorithms are applied on batches $B_{1: 4}$. Both of these update techniques operate at acceptance threshold set at $0.001 \%$ FAR (i.e., thr ${ }^{*}, t h r^{b 0} *$ and $t h r^{b 1} *$ in algorithms 1 and 2 ) which is same for all the users.

- After four updating cycles using batches $B_{1: 4}$, number of captured samples, belonging to subgraph containing enrolled templates and those captured from other different subgraphs are computed and presented in Figures 3(a) $3(\mathrm{~b})$ for each subject. These Figs. 3(a) 3(b) clearly show that co-update has the high potential to capture samples with significant variations (that is, belonging to different subgraphs) in contrary to self-update, using exactly the same acceptance threshold as that of self-update (0.001\% FAR).

- Finally, using the batch $B_{5}$, average mean genuine score over all the users is computed for each updating algorithm and the baseline classifier (without adaptation) for the templates updated till fourth update cycles. In case of baseline classifier, initial enrolled templates are matched against the batch $B_{5}$. The relative increase of the genuine mean score for self-update and coupdate techniques over the baseline classifier is computed to be $25 \%$ and $53 \%$, respectively. This is a direct confirmation of the increase in representativeness of the enrolled templates. 


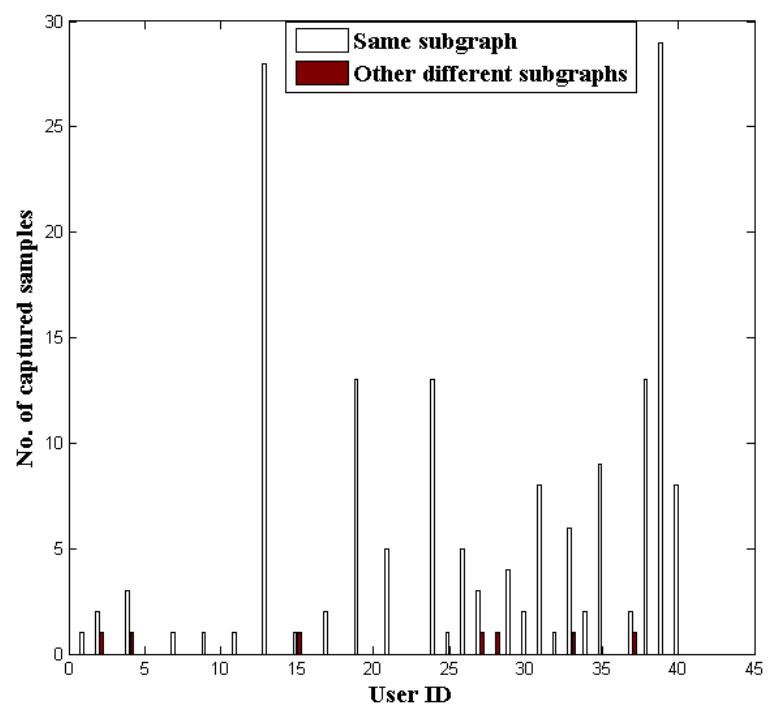

(a)

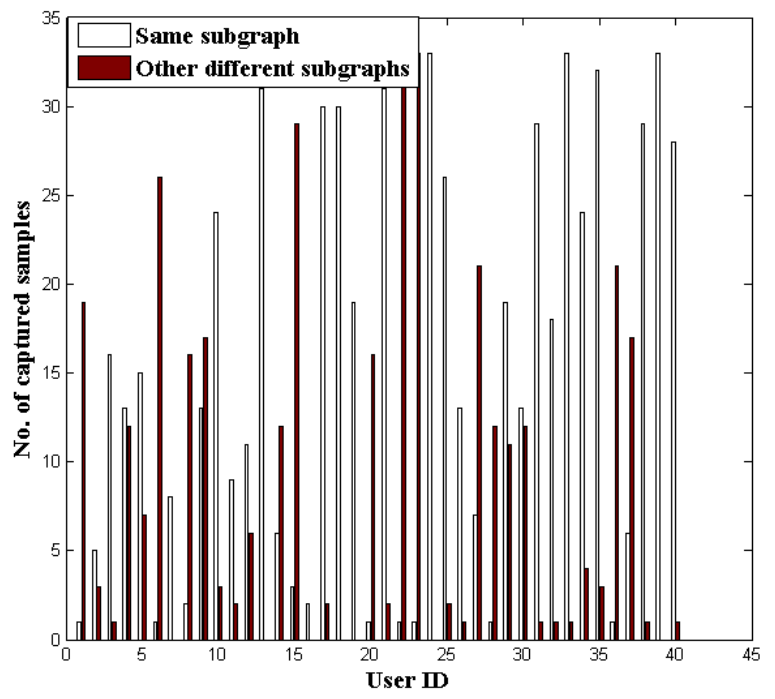

(b)

Fig. 3. Number of samples exploited by a) self-update and b)co-update process, from the same sub-graph to which the enrolled templates belong and from the other different sub-graphs. $X$ axis represent different users and $y$ axis represent number of captured samples from the same and different subgraphs. 
Table 1. Cumulative percentage of samples in the updated template set till each update cycle $i$ for the self-update and co-update process

\begin{tabular}{|c|c|c|c|c|}
\hline Update techniques & $(\%) B_{1}$ & $(\%) B_{1} \cup B_{2}$ & $(\%) B 1 \cup \ldots \cup B_{3}$ & $(\%) B 1 \cup \ldots \cup B_{4}$ \\
& & & 17 & 17 \\
\hline Self-update & 31 & 18 & 83 & 86 \\
\hline Co-update & 77 & 77 & \multicolumn{3}{|l|}{} \\
\hline
\end{tabular}

In addition to above evidences, Table 1, presents the cumulative percentage of samples after the $i-t h$ update cycle, that is, the total percentage of data from batches $B_{1} \cup \ldots \cup B_{i}$ which have been gradually added to the template gallery, again proving the efficacy of co-update.

\subsection{Experiment \#2}

The aim of the experiment is the performance assessment of the self-update and co-update in terms of Equal Error Rate $\left(E E R_{i}\right)$ as computed over time (see protocol). For the real time evaluation, five random impostor samples are also inserted in each batch $B_{i}$. Figures 4(a) and 4(b) show EER values for baseline, self- and co-update at each update cycle.

It can be seen that, over time, performance of the co-update significantly increases. Differences in performances are due to the intrinsic characteristic of face and fingerprint modalities, as may be deduced from both the figures. This is evident both for the face and fingerprint biometrics. Specifically, after the third update cycle, EER exhibits a decreasing trend. On the other hand, self-update strictly follows the trend of baseline matcher.

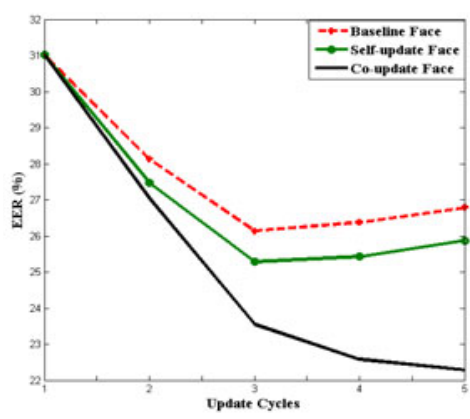

(a) Face

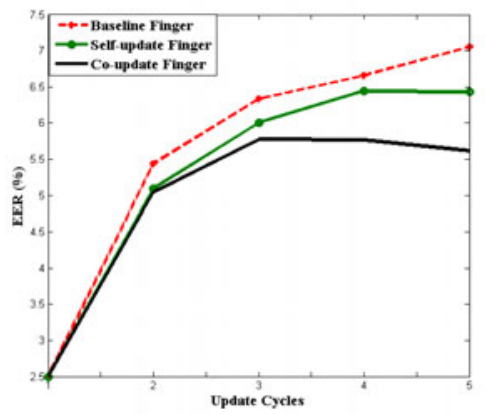

(b) Fingerprint

Fig. 4. EER Curve of the performance evaluation of the co-update in comparison to self-update and baseline classifier over time for a) face and b) fingerprint modalities 


\section{Conclusions}

In this paper, we experimentally evaluated the performance of self- and coupdate algorithms on multiple update cycles, available over time.

Existing claims in the literature such as: a) self-update is dependent on the threshold settings and on the representativeness of the initial templates, in capturing significant variations; on the contrary, co-update does not suffer from this limitation and b) self update process results in slow adaptation and limited performance improvement in comparison to co-update over time, were argued but never investigated.

We explicitly did such an analysis, which has been fully confirmed in our opinion, by the proposed conceptual representation supported by experimental evidences on DIEE Multi-modal data set.

Acknowledgments. This work has been partially supported by Regione Autonoma della Sardegna ref. no. CRP2-442 through the Regional Law n.7 for Fundamental and Applied Research, in the context of the funded project Adaptive biometric systems: models, methods and algorithms. Ajita Rattani is partly supported by a grant awarded to Regione Autonoma della Sardegna, PO Sardegna FSE 2007-2013, L.R. 7/2007 "Promotion of the scientific research and technological innovation in Sardinia".

\section{References}

1. Jain, A.K., Flynn, P., Ross, A.: Handbook of Biometrics. Springer, Heidelberg (2007)

2. Ross, A., Nandakumar, K., Jain, A.K.: Handbook of Multibiometrics. Springer, Heidelberg (2006)

3. Ling, H., Soatto, S., Ramanathan, N., Jacobs, D.: A study of face recognition as people age. In: Proc. 11th IEEE Int. Conf. on Computer Vision, Rio de Janeiro, Brazil, vol. 2688, pp. 1-8 (2007)

4. Rattani, A., Freni, B., Marcialis, G.L., Roli, F.: Template update methods in adaptive biometric systems: A critical review. In: Tistarelli, M., Nixon, M.S. (eds.) ICB 2009. LNCS, vol. 5558, pp. 847-856. Springer, Heidelberg (2009)

5. Roli, F., Marcialis, G.L.: Semi-supervised PCA-based face recognition using selftraining. In: Yeung, D.-Y., Kwok, J.T., Fred, A., Roli, F., de Ridder, D. (eds.) SSPR 2006 and SPR 2006. LNCS, vol. 4109, pp. 560-568. Springer, Heidelberg (2006)

6. Roli, F., Didaci, L., Marcialis, G.L.: Template co-update in multimodal biometric systems. In: Lee, S.-W., Li, S.Z. (eds.) ICB 2007. LNCS, vol. 4642, pp. 1194-1202. Springer, Heidelberg (2007)

7. Rattani, A., Marcialis, G.L., Roli, F.: Capturing arge intra-class variations of biometric data by template coupdate. In: Proc. of IEEE Computer Society Conference on Computer Vision and Pattern Recognition Workshops, Anchorage, Alaska, USA, pp. $1-6(2008)$

8. Diee multimodal database, http://prag.diee.unica.it/pra/eng/home 